\title{
Schritte zählen und Kinder zeugen für ein längeres Leben?
}

Chefredakteur Dr. med. Dirk Einecke

\section{WIEDERHOLTE MANDELENTZÜNDUNGEN}

\section{Wann lohnt die Tonsillektomie?}

Eine Adenotonsillektomie bei Kindern ist nur dann sinnvoll, wenn sie häufig an Halsschmerzen mit Schluckbeschwerden und Fieber erkranken (z. B. $\geq 7$ Episoden im letzten Jahr oder $\geq 5$ in jedem der letzten zwei Jahre) oder an Schlafapnoe leiden. Bei nur leichten bis mäßigen Beschwerden hat die Entfernung der Mandeln keinen klinisch relevanten Nutzen. Im Vergleich zu beobachtendem Abwarten reduziert die OP bei diesen Patienten Episoden mit Fieber und Halsentzündungen nur um 0,2 und Infekte der oberen Atemwege um 0,5 pro Personenjahr. Arch Otolar Head Neck Surg 2007;133:1083-88

\section{LUMIRACOXIB-ZULASSUNG RUHT}

\section{Verdacht auf Leberschäden}

Der selektive COX-2-Inhibitor Lumiracoxib (Prexige ${ }^{\circledR}$ ) darf in Deutschland vorläufig nicht verordnet werden. Anlass für die Entscheidung des BfArM sind Berichte über Leberschäden nach Anwendung des COX-2-Hemmers.

Bisher war man davon ausgegangen, dass nur höhere Dosierungen als in der

\section{DEPRESSIONEN UNTER RIMONABANT}

\section{Auch bei negativer Anamnese}

Schon vor einigen Monaten hat die amerikanische Zulassungsbehörde FDA auf ein erhöhtes Risiko für Depressionen und Suizidalität unter dem Cannabinoidantagonisten Rimonabant hingewiesen.

Diese Nebenwirkung bestätigt jetzt eine Metaanalyse der vier RIO-Studien mit 4100 Teilnehmern. Unter Rimonabant gab

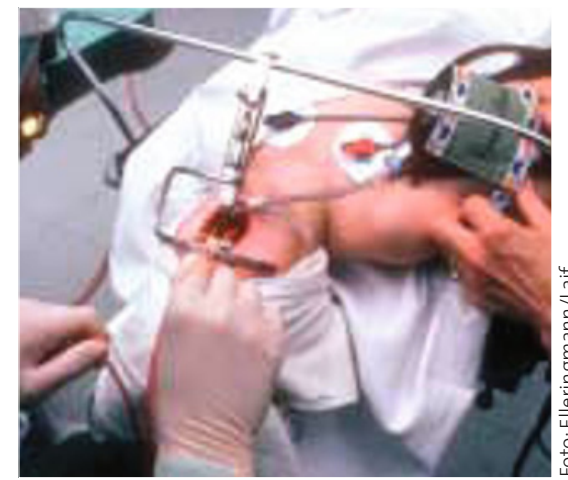

Bei nur mäßigen Beschwerden bringt die OP keine relevante Besserung.
EU zugelassen hepatotoxisch wirken können. Den neuen Daten zufolge können möglicherweise auch unter der in Deutschland zugelassenen Dosis von nur $100 \mathrm{mg}$ und bei kurzer Anwendungsdauer Leberschäden auftreten. Das Nutzen-Risiko-Verhältnis wird jetzt erneut überprüft. es mehr Therapieabbrüche wegen depressiver Störungen (3\% vs. $1,4 \%$ ) oder Angst ( $1 \%$ vs. 03\%). Die Daten zeigen, dass auch Patienten ohne psychiatrische Auffälligkeiten in der Anamnese unter dieser Therapie Depressionen entwickeln können. Adipöse Patienten neigen ohnehin dazu. Lancet 2007;370:1706-13

\section{Zitat der Woche}

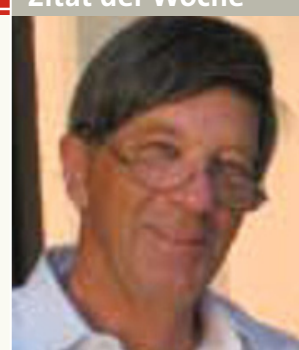

„Es gibt nichts Schöneres, als Arzt zu sein. Noch schöner aber ist es, Internist zu sein.“

Dr. Hartmuth Weinholz, Ehrenpräsident des Berufsverbands Deutscher Internisten (BDI), beim 1. Deutschen Internistentag am 27. Oktober 2007 in Berlin
NACH VENÖSER THROMBOEMBOLIE

\section{Erhöhtes Risiko für Herzinfarkt und Schlaganfall}

Patienten mit venöser Thromboembolie haben auch ein erhöhtes Risiko für Thrombosen im arteriellen Gefäßsystem. Besonders gefährdet sind sie im ersten Jahr nach einer tiefen Venenthrombose oder Lungenembolie: Herzinfarkte treten dann 1,6- bzw. 2,6-mal häufiger auf, Schlaganfälle 2,2- bzw. 2,9-mal so oft. Aber auch langfristig bleibt das kardiovaskuläre Risiko dieser Patienten erhöht, so das Ergebnis einer dänischen Registerstudie. Lancet 2007;370:1742-44

\section{MOTIVATION ZU MEHR BEWEGUNG}

\section{Mit Schrittzähler geht's besser}

Den inneren Schweinehund zu überwinden und körperlich aktiver zu sein, scheint vielen Menschen leichter zu fallen, wenn sie sich regelmäßig die Bilanz ihrer Bemühungen vor Augen führen können. Das ist das Ergebnis eines Reviews, der den Nutzen von Schrittzählern ausgewertet hat. Zuvor weitgehend inaktiven Personen gelang es damit, ihre tägliche Schrittzahl deutlich zu steigern. Gleichzeitig sanken BMI und Blutdruck. Besonders effektiv war die Maßnahme, wenn ein Schrittziel vorgegeben wurde. JAMA 2007;298:2296-2304

\section{GERONTOLOGIE}

\section{Kindersegen bringt Männern langes Leben}

In den Jahren 1917/18 wurden in den USA alle Männer bis 46 Jahre für den Militärdienst registriert. Diese Daten nutzten jetzt Gerontologen, um dem Geheimnis der Langlebigkeit auf die Spur zu kommen. Sie fanden 240 Männer vom Jahrgang 1887, die mindestens 100 Jahre alt geworden waren. Männer, die mit 30 schon ein bis drei Kinder in die Welt gesetzt hatten, hatten eine um 61\% größere Chance, $100 \mathrm{zu}$ werden, als kinderlose 30-Jährige. Ab dem vierten Kind verdreifachte sich die Wahrscheinlichkeit für extreme Langlebigkeit. Gerontological Society of America, 6oth Annual Scientific Meeting, San Francisco 16.-20. Nov. 2007 